\title{
Continuously Measurements of Energy Spectra of Cosmic-Ray-induced-neutrons on the Concordia Antarctic Station for the period 2015-2021
}

\author{
Guillaume HUBERT ${ }^{a, *}$ \\ ${ }^{a}$ ONERA DPHY, University of Toulouse \\ 2 av. Edouard Belin, Toulouse, France \\ E-mail: guillaume.hubert@onera.fr
}

The CHINSTRAP (Continuous High-altitude Investigation of the Neutron Spectra for Terrestrial Radiation Antarctic Project) supported by the French Polar Agency (IPEV) aims at recording cosmic-ray (CR) induced-neutron spectra at the Concordia station since December 2015. The neutron spectrometer measures the neutron spectrum over a wide energy range from meV up to tens of $\mathrm{GeV}$ with a short time resolution. Several parameters can influence the measurement, including systematic and environmental effects such as the atmospheric pressure, the hydrometric environment close to the instrument and the atmospheric water vapor. This paper presents CR induced neutrons measurements analyses from 2015 to 2021 in Concordia, integrating corrections to take into account environmental and systematic effects. Long-term and short-term analyses are proposed, applied to count rate, fluxes and spectra. A last part investigates the contribution of modelling to data analyses and the ability to deduce the solar modulation from neutron spectra and the radiation field extrapolation using nuclear transport in atmosphere. An underlying objective is also to improve physical models allowing analyses of continuous and simultaneously measurements of CR induced neutrons spectra.

37th International Cosmic Ray Conference (ICRC2021)

12-23 July 2021

Berlin, Germany - Online

"Speaker 


\section{Introduction}

The primary and the secondary radiation produced in the atmosphere can be a serious issue for the reliability of microelectronics devices embedded in aircraft [1] and this is of a major concern for aircrew member's dose assessment [2]. Moreover, Polar Region development induces an important issue related to space weather. Neutrons, protons and muons are the main secondary particles produced by the interaction of primary cosmic rays (CR) with the nuclei of the constituents of the atmosphere [3].

To study secondary CR at ground level, many detectors are distributed around the Earth, in particular in the Polar Regions. The Neutron Monitors (NMs) are used across the world to monitor CR in the vicinity of the Earth magnetosphere [4]. Another alternative is to use directly neutron spectrometry [5] technique which will make it possible to deduce the neutron energy spectrum. Among the most advanced systems, there are the Bonner Sphere Spectrometers (BSSs), based on spherical proportional counters [6]. Secondary particles are influenced by environmental and systematic effects [7]. CR flux increases at higher altitude as atmospheric air pressure and the shielding effect of the atmosphere decrease. Another effect concerns the albedo neutron produced by the interaction of air-shower neutrons with the surface of the Earth. Hydrogen in soil, air and snow determines the amount of ground albedo neutrons in the sensitive energy range from $1 \mathrm{eV}$ to $10 \mathrm{MeV}$. In order to get around the influence of these parameters, the detection of high energy neutrons (cascade neutron i.e. $\mathrm{E}>20 \mathrm{MeV}$ ) is preferred because environmental effects have a less impact.

The CHINSTRAP (Continuous High-altitude Investigation of the Neutron Spectra for Terrestrial Radiation Antarctic Project) supported by the French Polar Agency (IPEV) aims at recording neutron spectra at the Concordia station since December 2015 [8]. Moreover, water vapor contents were recorded in Concordia station in the framework of the HAMSTRAD (H2O Antarctica Microwave Stratospheric and Tropospheric Radiometers) project [9]. Temporal series of meteorological data allow taking into account corrections in the CR analyses. This paper proposes to present CR-induced-neutrons measurements analyses from 2015 to 2021 taking into account environmental and systematic effects. The contribution of modelling to data analyses, in particular the ability to deduce the solar modulation from neutron spectra and the radiation field extrapolation using nuclear transport in atmosphere is finally studied.

\section{Description of the CHINSTRAP and HAMSTRAD projects}

The Concordia station is a key location for the study of CR because of its geomagnetic conditions $(\sim 0.001 \mathrm{GV})$ and its high altitude $(+3233 \mathrm{~m}$ above the sea level). And this is especially true for observing solar particle events such as Forbush Decreases (FD) or Ground Level Enhancement (GLE). In the framework of their research activities, the Concordia station benefits from two research programs, which although developed in a completely compartmentalized way, ended up converging towards a common interest. In chronological order, the first is the HAMSTRAD [9] project of the aim is to investigate since 2012 the trends in water vapor and temperature profiles from the lower part of the troposphere to the lower part of the stratosphere. The second is the CHINSTRAP [8] project aims to continuously measure 
the atmospheric neutron spectra since December 2015. The scientific instruments used in the framework of these two projects are installed in the shelter named "Physics" and located one kilometer from the station.

\section{Impacts of Environmental and systematic effects}

Fig. 1 presents temporal series of the LWP, the IWV and the atmospheric air pressure measured in Concordia station from 2016 to 2021. LWP and IWV are issued from the radiometer, while the atmospheric pressure is directly extracted by using a pressure detector integrated in the neutron spectrometer system. Winter / summer cycles are very clearly observed on the three parameters, that is to say summers (December, January and February) which are characterized by high atmospheric air pressures and higher humidity (liquid and vapor) and conversely in winters (June, July and August). Concordia appears as one of the driest and coldest places over the world with precipitable water or Integrated Water Vapor (IWV) less than $1 \mathrm{~kg} \mathrm{~m}-2$ over the year reaching less than $0.1 \mathrm{~kg} \mathrm{~m}-2$ in winter [10]. The atmosphere above Dome $\mathrm{C}$ as dry when IWV is less than $1.0 \mathrm{~kg} . \mathrm{m}-2$, which is very low compared to values observed in typical moist conditions (i.e. $20 \mathrm{~kg} . \mathrm{m}-2$ at middle latitudes). Note that liquid water (LPW) is a factor 102-103 less intense than water vapour (IWV) above Dome C.

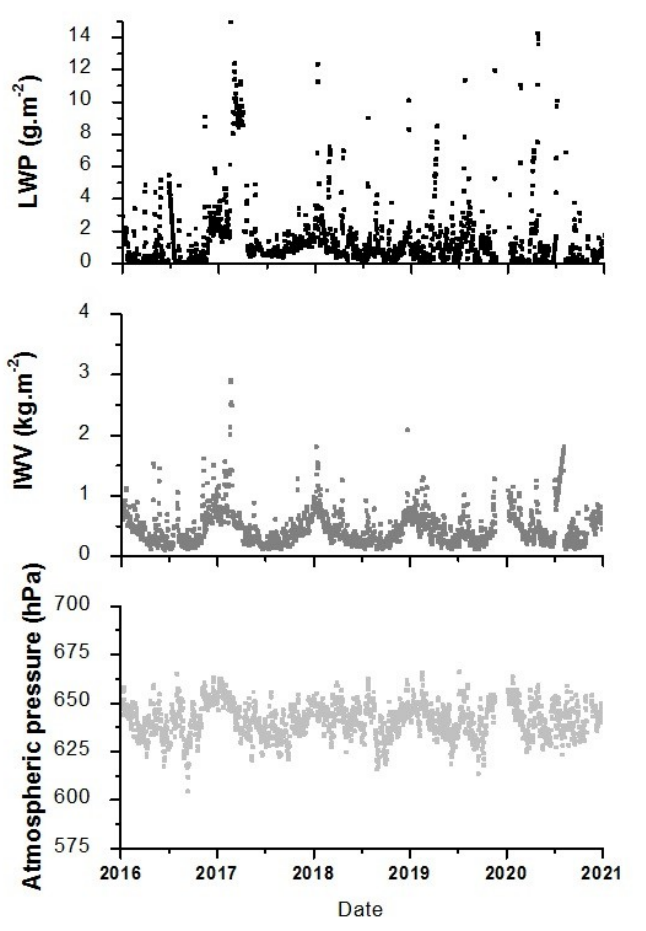

Fig. 1 - Temporal evolution of the daily LWP (g.m-2) and IWV (kg.m-2) as measured by HAMSTRAD above the altitude of Concordia station from January 2016 to January 2021. Also shown is the daily atmospheric pressure measured in Concordia during the same period thanks to the pressure detector integrated in the neutron spectrometer.

The atmospheric air pressure varies from 600 to $665 \mathrm{hPa}$, which implies a substantial modification of the atmospheric structure, and consequently a significant impact on the production of secondary particles. 


\section{Long-term analyses of Cosmic-Ray-induced-neutrons}

\subsection{Analyses of neutron spectra}

The CR neutron energy spectra can be obtained by unfolding the measured count rates and using response functions. The neutron spectrum measured by Goldhagen et al. was employed as an initial guess spectrum for unfolding.
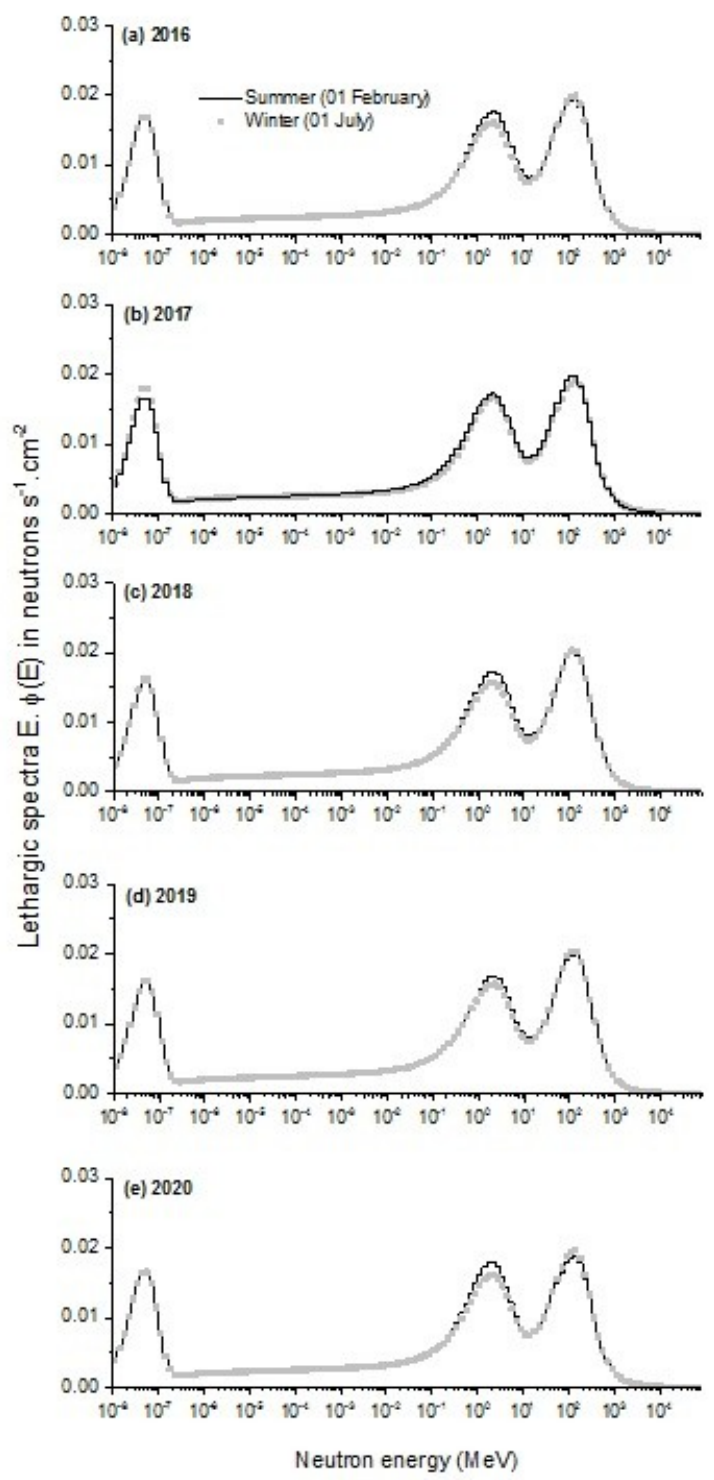

Fig. 2 - Neutron spectra in lethargic representation measured in Concordia each 01 February and 01 July of the years 2016, 2017, 2018, 2019 and 2020. Count rates used for the unfolding process were corrected for the atmospheric pressure and to the water vapor content. Moreover, each spectrum was calculated by considering an integration time of 1 day.

Fig. 2 presents a sample of neutron spectra in lethargic representation (i.e. the product of the neutron energy to the differential spectrum). Spectra were measured in Concordia each beginning of February and July months of the years 2016, 2017, 2018, 2019 and 2020. It is useful to remember that the months of February and July correspond to summer and winter in 
the southern hemisphere, respectively. Count rates used for the unfolding process were corrected to the atmospheric pressure and to the water vapor content. Moreover, each spectrum was calculated with an integration time of 1 day.

The total energetic spectral distribution can be divided in four regions. The thermal region characterized by neutron energy from $1 \mathrm{meV}$ to $0.5 \mathrm{eV}$. From $0.5 \mathrm{eV}$ to $0.1 \mathrm{MeV}$, there is the epithermal plateau and at high energies, there are two peaks, the evaporation peak from $0.1 \mathrm{MeV}$ to $20 \mathrm{MeV}$ and the cascade one above $20 \mathrm{MeV}$. It is interesting to note that the spectra resulting from the summer measurements (i.e. February) have a greater evaporation component than those carried out in winter (i.e. July), and this is globally verified for each year. Since barometric and water vapor corrections have been considered, the most plausible explanation is the more important presence of liquid water in summer than in winter as shown in Fig. 3 for the LWP parameter. Another remark is that the thermal domain does not vary with season. This is an atypical behavior in comparison with data from other stations (i.e. in the Pic du Midi and in the Pico dos Dias observatories), and which is undoubtedly the consequence of the invariance of the soil's hydric environment. It seems that the cascade neutron peak is higher in summer than in winter, which is consistent with the observations and hypotheses discussed for the evaporation peaks.

\subsection{Analyses of neutron fluxes}

The neutron flux derived from the unfolding of the daily averaged count rates in Concordia are presented in Fig. 4 from 2016 to 2021. The total neutron flux seems to increase, which is consistent with the dynamics of the solar activity, i.e. the decreasing part of the solar cycle. Orders of magnitude are from 0.16 to 0.18 neutron cm-2.s-1.
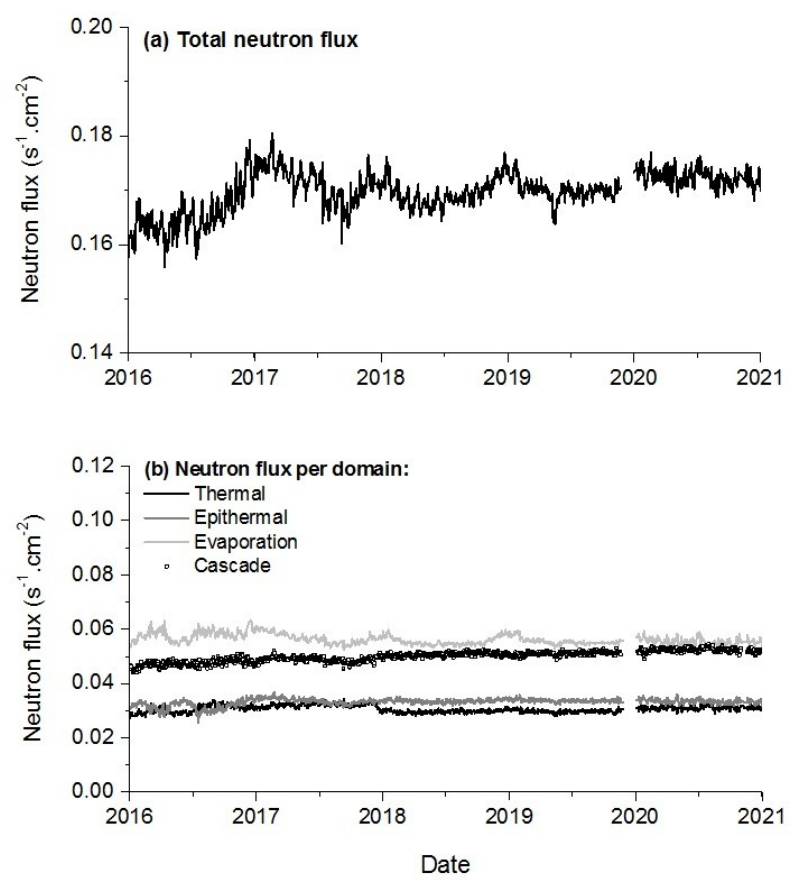

Fig. 3 - Integrated fluence rates derived from the unfolding of the six hour averaged count rates in the Concordia station from January 2016 to 2021. Results present (a) the total and the cascade energy domain contributions, (b) the evaporation, the epithermal and the thermal energy domain contributions. 
Contrary to data issued from high altitude (i.e. Pic-du-Midi, Pico dos Dias Observatory), temporal oscillations were not observed, neither for the total neutron flux, nor for the different energy domains. A slight oscillation of evaporation neutron fluxes correlated with the winter / summer cycle is observed.

\section{Contribution of modeling to data analyzes}

The aim of the ATMOS-CORE platform [11] can be declined according to four fields of interest: 1) the computation of atmospheric secondary $\mathrm{CR}$ spectra (neutrons, protons, muons and electrons) in combining Geant4 simulations of extensive Air Showers (EAS) and a primary GCR spectra defined by the Force-Field Approximation, 2) the assessment of the Single Event Risk [12][13], 3) the assessment of the ambient dose equivalent and 4) the calculation of the cosmogenic isotope production. The calculation of the secondary CR spectra is clearly the keystone of the simulation flow, and only this part concerns analyzes proposed by this work. The neutron energetic spectrum allows accessing to CRs properties enriched in comparison to NMs. This offers an opportunity for another method to reconstruct the time series of the solar modulation.

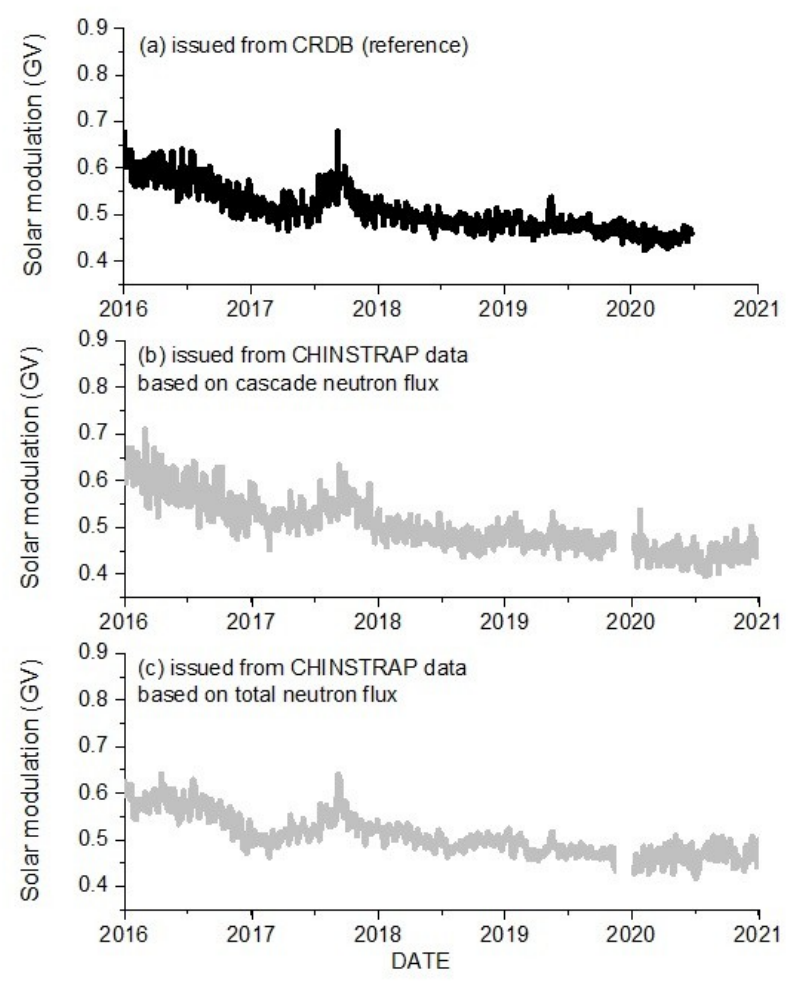

Fig. 4 - Solar modulation from 2016 to 2021 deduced from (a) the Cosmic-Ray DataBase, (b) the neutron cascade calculated from CHINSTRAP records and (c) the total neutron flux calculated from CHINSTRAP records. The integration time is considered as 1 day.

The solar modulation can be also extracted using neutron spectra and fluxes issued from CHINSTRAP data. An iterative method determines the solar modulation value for which the calculated cascade neutron fluxes are equivalent to the ones deduced from CHINSTRAP data. 
The geographic and cutoff rigidity characteristics of the Concordia station are the other input parameters of the calculations (i.e. $+3233 \mathrm{~m}, \sim 0 \mathrm{GV}$ ).

Fig. 4 presents the solar modulation from January 2016 to January 2021 deduced from (a) the Cosmic-Ray DataBase (CRDB, [14]), (b) the neutron cascade calculated from CHINSTRAP records and (c) the total neutron flux calculated from CHINSTRAP records. The CRDB provides the solar modulation from NM data, allowing to calculate top-of-atmosphere fluxes from interstellar (fluxes for comparisons to GCR data). The procedure and calibration to extract $f(t)$ from various NM stations are detailed in [15].

Solar modulation values extracted from both methods are consistent to each other, with in particular a peak observed around the end of winter 2017 (September). It seems all the same that the temporal resolution is better for the data extracted from NMs whose sensitivities are higher than that of the neutron spectrometer. However, accessing the shape of the energy spectrum over a thermal-cascade energy range partially compensates for the negative impact of lower sensitivity. It is very interesting to note that calculations based on the total neutron flux achieve really satisfactory results, with a resolution that seems as good as those issued from the CRDB. This is a new result as usually (i.e. in high-altitude observatories located at medium latitudes) it is not possible to extract the solar potential in considering the whole neutron energy range due to environmental effects on low energies.

\section{Conclusion}

In this paper, we have presented analyses of the atmospheric neutron spectra and fluxes recorded in the Concordia station from 2015 to 2021 recorded in the framework of the CHINTRAP project. The Concordia station also benefits from the HAMSTRAD project, providing water vapor profiles from the lower part of the troposphere to the lower part of the stratosphere. Data issued from the radiometer and atmospheric pressure detector allow to apply a correction factor which reflects the impact of the systematic and environmental effects.

The analysis of neutron spectra shows that spectra resulting from the summer measurements (i.e. February) have a greater evaporation component than those carried out in winter (i.e. July), and this is globally verified for each year. Since barometric and water vapor corrections have been considered, the most plausible explanation is the more important presence of liquid water in summer than in winter. This is a simple hypothesis that needs to be supported by accumulating additional measurements over a very long period.

The cascade neutron is least affected by the local environment and it is possible to deduce the solar modulation in using Concordia data. This result shows that neutron spectrum can be quantitatively correlated using modeling and cascade neutron. Moreover, results highlight that the Concordia station is an exceptional site to study the neutron spectrum because the entire energy spectrum is usable, provided that the corrections related to atmospheric air pressure and the water vapor content are applied. Indeed, results have shown clearly the solar modulation extracted from the total neutron flux allows to obtain a better resolution than that obtained with other methods (with an equivalent integration time). 


\section{Acknowledgments}

The authors thank the personnel of the Concordia station for their great help and hard work in harsh conditions. This work is supported by the IPEV (French Polar Institute, Institut PaulEmile Victor) in the framework of the CHINSTRAP project.

\section{References}

[1] P. Peronnard, R. Velazco and G. Hubert, "Real-life SEU experiments on 90nm SRAMs in Atmospheric Environment: measures vs. predictions done by means of MUSCA SEP3 platform", Nuclear Science, IEEE Transactions on, 56(6):3450 - 3455, 2009.

[2] G. Reitz, "Radiation environment in the stratosphere. Radiat Prot Dosimetry", 1993; 48(1):5-20.

[3] P. K. F. Grieder, "Cosmic Rays at Earth: Researcher's Reference Manual and Data Book" 1st ed., pp. 1093, Elsevier, 2001.

[4] C. Lopade, Space physics data system climax and haleakala neutron monitor datasets: neutron monitor stations, Univ. of N. H. Durham, (available at

http://ulysses.sr.unh.edu/NeutronMonitor/Stations.txt), 2009.

[5] P. Goldhagen, "Cosmic-ray neutrons on the ground and in the atmosphere", MRS Bulletin, 28(2):131 (2003).

[6] A. Cheminet, V. Lacoste, V. Gressier, G. Hubert, A. Martin and M. Pépino, "Characterization of the IRSN neutron multisphere spectrometer (HERMEIS) at European standard calibration fields", IOP Science Journal of Instrumentation, 2nd FNDA, Vol. 7, 2012.

[7] R. Rosolem, Shuttleworth, W. J., Zreda, M., Franz, T. E., Zeng, X., Kurc, S. A. (2013). The effect of atmospheric water vapor on neutron count in the cosmic-ray soil moisture observing system, Journal of Hydrometeorology, 14, 1659-1671.

[8] G. Hubert, Pazianotto, M. T., Federico, C. A., "Modeling of ground albedo neutrons to investigate seasonal cosmic-ray-induced neutron variations measured at high-altitude stations", J. Geophys. Res. Space Physics, 121, 186-201, 2016. 